\title{
ON DOMINATED EXTENSIONS IN FUNCTION ALGEBRAS ${ }^{1}$
}

\author{
J. GLOBEVNIK
}

\begin{abstract}
The Bishop-Gamelin interpolation theorem asserts that given a compact Hausdorff space $K$, a closed subspace $A$ of $C(K)$, a positive continuous function $p$ on $K$ and a closed set $F \subset K$ such that every measure in the annihilator of $A$ vanishes on $F$, every function $f \in C(F)$ satisfying $|f(s)|<p(s)(s \in F)$ extends to a function $\tilde{f} \in A$ satisfying $|\tilde{f}(z)|<p(z)(z \in K)$. In the paper we consider a special case where the theorem is extended to the situation when the dominating function is nonnegative.
\end{abstract}

Let $K$ be a compact Hausdorff space and $A$ a closed subspace of $C(K)$, the space of all (real- or complex-valued) continuous functions on $K$, with sup norm. Let $A^{\perp}$ denote the set of all annihilating measures of $A$ and $\mu_{F}$ the restriction of the measure $\mu$ to $F$, a closed subset of $K$. If $\mu \in A^{\perp}$ implies that $\mu_{F}=0$ then

given any positive continuous function $q$ on $K$ and any $f \in C(F)$ satisfying $|f| \leqslant q$ on $F$ there is some $\tilde{f} \in A$ which extends $f$ and satisfies $|\tilde{f}| \leqslant q$ on $K$.

This is a strengthened form of a well-known theorem of E. Bishop [2] which is a special case of a more general theorem proved by T. W. Gamelin [3]. Note that (1) implies (1) with $<$ replaced by $<$.

In known generalizations and applications of Bishop's theorem [1], [3], [4], [5], [6], [7], [9], [10], [11] the dominating functions $q$ are always assumed to be positive on $K$. Our purpose here is to present a special case where the conclusion of Bishop's theorem can be strengthened to allow domination by nonnegative functions.

Let $K, F$ and $A$ be as above and let $p$ be a nonnegative continuous function on $K$. Is it true that

given any $f \in C(F)$ which satisfies $|f|<p$ on $F$ there exists an

$\tilde{f} \in A$ which extends $f$ and satisfies $|\tilde{f}| \leqslant p$ on $K$ ?

We begin with two examples. Let $T$ be the unit circle in $\mathrm{C}$ and let $B \subset C(T)$ be the disc algebra. By the $\mathrm{F}$. and $\mathrm{M}$. Riesz theorem any closed set $F \subset T$ of Lebesgue measure 0 satisfies the condition $\mu \in B^{\perp} \Rightarrow \mu_{F}=0$ [8]. Assume that $p$ is a nonnegative continuous function on $T$ which vanishes on a set of positive Lebesgue measure on $T$ but does not vanish identically. Then any $g \in B$ satisfying $|g| \leqslant p$ on $T$ vanishes on a set of positive Lebesgue measure and consequently

Received by the editors March 23, 1979 and, in revised form, June 5, 1979.

AMS (MOS) subject classifications (1970). Primary 46J10.

'This work was supported in part by the Boris Kidric Fund, Ljubljana, Yugoslavia.

(c) 1980 American Mathematical Society 0002-9939/80/0000-0364/\$02.50 
vanishes identically [8]. This shows that (2) is false in general. If (2) is satisfied then clearly

for each $x \in F-p^{-1}(0)$ there is some $g \in A$ which satisfies $g(x) \neq 0$ and $|g| \leqslant p$ on $K$.

Suppose that (3) is satisfied. Let $A \subset C(T)$ be the subspace spanned by $B$ and a function $h \in C(T)-B$ which vanishes on a set of positive Lebesgue measure but does not vanish identically. Again, since $B \subset A$, any closed set $F \subset T$ of Lebesgue measure 0 satisfies the condition $\mu \in A^{\perp} \Rightarrow \mu_{F}=0$. Put $p(z)=|h(z)|(z \in T)$. Then $A$ and $p$ satisfy (3). Suppose that $g \in A$ satisfies $|g|<p$ on $T$. Then $g$ vanishes on the zero set of $h$ and consequently $g$ is a scalar multiple of $h$. This shows that (3) is not a sufficient condition for (2) if $A$ is a subspace of $C(K)$. However, if $A$ is a subalgebra of $C(K)$ then (3) implies (2) and this is the main result of the present paper.

If $A$ and $F$ satisfy (1) then we will say that $A$ has the dominated extension property with respect to $F[\mathbf{1 0}]$.

TheOREM. Let $K$ be a compact Hausdorff space and let $A \subset C(K)$ be a closed subalgebra. Suppose that $F \subset K$ is a closed set such that $A$ has the dominated extension property with respect to $F$. Let $p$ be a nonnegative continuous function on $K$ such that for any $x \in F-p^{-1}(0)$ there is some $v \in A$ satisfying $v(x) \neq 0$ and $|v(z)|<p(z)(z \in K)$. Then given any $f \in C(F)$ satisfying $|f(s)|<p(s)(s \in F)$ there is an extension $\tilde{f} \in A$ of $f$ such that $|\tilde{f}(z)|<p(z)(z \in K)$.

LEMMA. Let $K$ be a compact Hausdorff space, $A \subset C(K)$ a closed subalgebra and $G \subset K$ a closed set such that $A$ has the dominated extension property with respect to $G$. Let $p$ be a nonnegative continuous function on $K$ such that given any $x \in G$ there is some $v \in A$ satisfying $v(x) \neq 0$ and $|v(z)|<p(z)(z \in K)$. Then given any neighbourhood $U$ of $G$ there is a function $h \in A$ such that

(i) $\|h\|=1$,

(ii) $h(s)=1(s \in G)$,

(iii) $|h(z)|<p(z)(z \in K-U)$.

Proof. Assume for a moment that some $g \in A$ satisfies $g(z) \neq 0(z \in G)$ and $|g(z)|<p(z)(z \in K)$. Let $U$ be a neighbourhood of $G$. Passing to a smaller $U$ if necessary we may assume with no loss of generality that $U$ is open and that $1 /|g|$ is bounded on $U$. By the Urysohn lemma there is some $\Phi \in C(K)$ such that $0<\Phi<1, \Phi \mid G=1$, and $\Phi \mid(K-U)=0$. Then

$$
z \rightarrow \phi(z)=\left\{\begin{array}{l}
\Phi(z) /|g(z)| \quad(z \in U), \\
0 \quad(z \in K-U),
\end{array}\right.
$$

is a nonnegative continuous function on $K$. Choose $\eta$ : $0<\eta<1$ so that $\eta \cdot\|g\|<$ 1 and let $\psi(z)=\max \{\eta, \phi(z)\}(z \in K)$. The function $\psi$ is positive and continuous on $K$ and satisfies $\psi(z)<1 /|g(z)|(z \in U)$. If $z \in G$ then $\psi(z)=\max \{\eta, \phi(z)\}=$ $\max \{\eta, 1 /|g(z)|\}=1 /|g(z)|$ so $|1 / g(z)|<\psi(z) \quad(z \in G)$. Since $A$ has the dominated extension property with respect to $G$ there is some $u \in A$ satisfying 
$u(z)=1 / g(z)(z \in G)$ and $|u(z)|<\psi(z)(z \in K)$. Define $h=u \cdot g$. Then $h$ belongs to $A$ and satisfies (ii). If $z \in U$ then $|h(z)|<\psi(z) \cdot|g(z)|<(1 /|g(z)|) \cdot$ $|g(z)|=1$. Let $z \in K-U$. Then $\phi(z)=0$ so $\psi(z)=\eta$. Consequently $|h(z)|<\psi(z)$ $\cdot|g(z)|=\eta \cdot|g(z)|$. Since $\eta<1$ it follows that $|h(z)|<|g(z)|<p(z)$ which proves (iii). Since $\eta \cdot\|g\|<1$ we also have $|h(z)|<1$ which completes the proof of (i).

It remains to show that there is some $g \in A$ such that $g(z) \neq 0(z \in G)$ and $|g(z)|<p(z)(z \in K)$. Clearly there is a neighbourhood $V$ of $G$ such that $p(z)>\varepsilon$ $(z \in V$ ) for some $\varepsilon>0$. By the assumptions and by the compactness of $G$ there are $v_{1}, v_{2}, \ldots, v_{n} \in A,\left|v_{i}(z)\right| \leqslant p(z)(z \in K)$, and positive numbers $\eta_{1}, \eta_{2}, \ldots, \eta_{n}$ such that $U_{i}=\left\{x \in G:\left|v_{i}(x)\right|>\eta_{i}\right\}(1 \leqslant i<n)$ are nonempty (relatively) open subsets of $G$ which cover $G$. Let $\left\{\Phi_{i}\right\}$ be a subordinated partition of unity on $G$. By the properties of $G$ each $\Phi_{i}$ has an extension $\tilde{\Phi}_{i} \in A$. Define the closed sets $G_{i} \subset G$ by $G_{i}=\left\{x \in G:\left|v_{i}(x)\right| \geqslant \eta_{i}\right\} \quad(1<i \leqslant \eta)$. Since $A$ has the dominated extension property with respect to $G$ it has the dominated extension property with respect to every closed subset of $G$, in particular, with respect to $G_{i}(1<i<n)$. By the first part of the proof there are functions $h_{i} \in A(1<i<n)$ satisfying $\left\|h_{i}\right\|=1$, $h_{i} \mid G_{i}=1$ and $\left\|\tilde{\Phi}_{i}\right\| \cdot\left|h_{i}(z)\right|<p(z) / n(z \in K-V)$. Since $U_{i} \subset G_{i}(1<i<n)$ it follows that $h_{i}(z) \tilde{\Phi}_{i}(z)=\Phi_{i}(z)(z \in G, 1<i<n)$. Define $u=\sum_{i=1}^{n} \tilde{\Phi}_{i} \cdot h_{i}$. Clearly $u \mid G=1$. If $z \in K-V$ then $|u(z)|<\sum_{i=1}^{n}\left|\tilde{\Phi}_{i}(z)\right| \cdot\left|h_{i}(z)\right|<n \cdot(p(z) / n)=p(z)$. It follows that $g=\min \{1, \varepsilon /\|u\|\} \cdot u$ has all the required properties. Q.E.D.

Proof of Theorem. Denote by $\mathbf{N}$ the set of all nonnegative integers. The case $f=0$ is trivial so with no loss of generality assume that $\sup _{s \in F}|f(s)|=1$ and $|f(s)|<p(s)(s \in F)$. Then $F_{n}=\left\{z \in F:|f(z)|>1 / 2^{n}\right\}$ is an increasing sequence of nonempty closed subsets of $F$ disjoint from $p^{-1}(0)$. By the Urysohn lemma there exist $\Phi_{n} \in C(F)(n \in \mathbf{N})$ such that for each $n \in \mathbf{N}$

$$
\begin{aligned}
\Phi_{n}(z) & =1-1 / 2^{n} \quad\left(z \in F_{n}\right), \\
0 & <\Phi_{n}(z)<1-1 / 2^{n} \quad(z \in F), \\
\Phi_{n}(z) & =0 \quad\left(z \in F-F_{n+1}\right) .
\end{aligned}
$$

Note that the sequence $\Phi_{n}$ is increasing. Put $f_{n}(z)=\Phi_{n}(z) f(z)(z \in F, n \in \mathbf{N})$. Clearly $f_{n} \in C(F)(n \in N)$ and by the properties of $\Phi_{n}$ we have

$$
\lim _{n \rightarrow \infty} f_{n}(s)=f(s) \quad(s \in F) .
$$

Let $n \in \mathbf{N}$. If $z \in F_{n}$ then

$$
\begin{aligned}
\left|f_{n+1}(z)-f_{n}(z)\right| & =\left|\Phi_{n+1}(z)-\Phi_{n}(z)\right| \cdot|f(z)| \\
& \leqslant\left|\Phi_{n+1}(z)-\Phi_{n}(z)\right|=1 / 2^{n+1} .
\end{aligned}
$$

If $z \in F-F_{n}$ then $|f(z)|<1 / 2^{n}$ so

$$
\left|f_{n+1}(z)-f_{n}(z)\right|=\left|\Phi_{n+1}(z)-\Phi_{n}(z)\right| \cdot|f(z)|<1 / 2^{n} .
$$

It follows that

$$
\left|f_{n+1}(s)-f_{n}(s)\right|<1 / 2^{n} \quad(s \in F, n \in \mathbf{N})
$$


Suppose that we have constructed a sequence $\tilde{f}_{i} \in A(i \in \mathbf{N})$ such that for each $i \in \mathbf{N}$
(a) $\tilde{f}_{i} \mid F=f_{i}$,
(b) $\left\|\tilde{f}_{i+1}-\tilde{f}_{i}\right\|<1 / 2^{i}$,
(c) $\left|\tilde{f}_{i}(z)\right|<\left(1-1 / 2^{i+1}\right) p(z)\left(z \in K-p^{-1}(0)\right)$,
(d) $\tilde{f}_{i}(z)=0\left(z \in p^{-1}(0)\right)$.

Define $\tilde{f}(z)=\lim \tilde{f}_{i}(z)(z \in K)$. Since $\tilde{f}_{i} \in A(i \in \mathbf{N})$ and since $A$ is closed it follows by (b) that $\tilde{f} \in A$. By (a) and (4) $\tilde{f}$ extends $f$. By (c) and (d) we have $|\tilde{f}(z)| \leqslant p(z)(z \in K)$.

It remains to prove the existence of $\tilde{f}_{i}$ satisfying (a) through (d) above. Put $\tilde{f}_{0}=0$. Clearly $\tilde{f}_{0}$ satisfies (a), (c) and (d) for $i=0$. Let $n \in \mathbf{N}$ and suppose that we have constructed $\tilde{f}_{n} \in A$ satisfying (a), (c) and (d) for $i=n$. By (c) we have

$$
\left(1-1 / 2^{n+2}\right) p(z)-\left|\tilde{f}_{n}(z)\right|>0 \quad\left(z \in K-p^{-1}(0)\right) \text {. }
$$

Since the term on the left is continuous in $z$ and since $F_{n+2}$ is a compact set disjoint from $p^{-1}(0)$ it follows that there is some $\eta>0$ and a neighborhood $U$ of $F_{n+2}$ such that

$$
\left(1-1 / 2^{n+2}\right) p(z)-\left|\tilde{f}_{n}(z)\right|>\eta \quad(z \in U)
$$

Define

$$
\phi(z)=\max \left\{\left(1-1 / 2^{n+2}\right) p(z)-\left|\tilde{f}_{n}(z)\right|, \eta\right\} \quad(z \in K) .
$$

Clearly $\phi$ is a positive continuous function on $K$. Let $s \in F_{n+2}$. Since $p(s)>0$ it follows that

$$
\begin{aligned}
\left|f_{n}(s)\right|+\left|f_{n+1}(s)-f_{n}(s)\right| & =\Phi_{n}(s) \cdot|f(s)|+\left[\Phi_{n+1}(s)-\Phi_{n}(s)\right] \cdot|f(s)| \\
& =\Phi_{n+1}(s) \cdot|f(s)| \leqslant\left(1-1 / 2^{n+1}\right) p(s) \\
& <\left(1-1 / 2^{n+2}\right) \cdot p(s)
\end{aligned}
$$

and consequently $\left|f_{n+1}(s)-f_{n}(s)\right|<\phi(s)\left(s \in F_{n+2}\right)$. If $s \in F-F_{n+2}$ then $f_{n}(s)=$ $f_{n+1}(s)=0$ so $\left|f_{n+1}(s)-f_{n}(s)\right|<\phi(s) \quad\left(s \in F-F_{n+2}\right)$. Define $\psi(z)=$ $\min \left\{1 / 2^{n}, \phi(z)\right\}(z \in K)$. Clearly $\psi$ is a positive continuous function on $K$. By (5) and by the preceding discussion it follows that $\left|f_{n+1}(s)-f_{n}(s)\right|<\psi(s)(s \in F)$. By the dominated extension property of $A$ with respect to $F$ there is some $u \in A$ such that

$$
u(s)=f_{n+1}(s)-f_{n}(s) \quad(s \in F)
$$

and

$$
|u(z)|<\psi(z) \quad(z \in K)
$$

which gives

$$
|u(z)|<\phi(z) \quad(z \in K)
$$

and

$$
\|u\|<1 / 2^{n}
$$

If $u=0$ then put $\tilde{f}_{n+1}=\tilde{f}_{n}$ and we are done. Assume that $u \neq 0$. Since $A$ has the dominated extension property with respect to $F$ it has the dominated extension 
property with respect to $F_{n+2}$. By lemma it follows that there is some $h \in A$ which satisfies

$$
\begin{gathered}
\|h\|=1, \\
h \mid F_{n+2}=1, \\
|h(z)| \leqslant p(z) /\left(2^{n+2} \cdot\|u\|\right) \quad(z \in K-U) .
\end{gathered}
$$

Define $\tilde{f}_{n+1}=\tilde{f}_{n}+h \cdot u$. Clearly $\tilde{f}_{n+1} \in A$. By (7) and (11) we have $(h u) \mid F_{n+2}=$ $\left(f_{n+1}-f_{n}\right) \mid F_{n+2}$. Since $\left(f_{n+1}-f_{n}\right) \mid\left(F-F_{n+2}\right)=0$ and since $\tilde{f}_{n} \mid F=f_{n}$ it follows that (a) is satisfied for $i=n+1$. By (9) and (10) (b) also is satisfied for $i=n$. Observe that $p(z)>0(z \in U)$ so, by (12), $h(z)=0\left(z \in p^{-1}(0)\right)$. Since $\tilde{f}_{n}(z)=0$ $\left(z \in p^{-1}(0)\right)$ it follows that (d) is satisfied for $i=n+1$. Let $z \in U$. By (6) we have $\phi(z)=\left(1-1 / 2^{n+2}\right) p(z)-\left|\tilde{f}_{n}(z)\right|$ which by (8) implies that $|u(z)|<$ $\left(1-1 / 2^{n+2}\right) p(z)-\left|\tilde{f}_{n}(z)\right|$. By $(10)$ it follows that $\left|\tilde{f}_{n+1}(z)\right|<\left|\tilde{f}_{n}(z)\right|+|u(z)|<$ $\left(1-1 / 2^{n+2}\right) p(z)$ which proves (c) for $z \in U$ and $i=n+1$. Finally, let $z \in$ $\left(K-p^{-1}(0)\right)-U$. By (12) and by (c) for $i=n$ it follows that

$$
\begin{aligned}
\left|\tilde{f}_{n+1}(z)\right| & \leqslant\left|\tilde{f}_{n}(z)\right|+|h(z)| \cdot|u(z)| \\
& \leqslant\left(1-1 / 2^{n+1}\right) p(z)+|u(z)| \cdot p(z) /\left(2^{n+2} \cdot\|u\|\right) \\
& \leqslant\left(1-1 / 2^{n+2}\right) p(z)
\end{aligned}
$$

which completes the proof of (c) for $i=n+1$. Q.E.D.

REMARK 1. The theorem still holds if we assume that $p$ is lower semicontinuous. Namely, (1) holds for all positive continuous functions $q$ iff it holds for all positive lower semicontinuous functions [10] so our proofs work also in the case when $p$ is lower semicontinuous.

REMARK 2. By changing slightly the proofs of the lemma and theorem one can prove the following: Let $K, A$ and $F$ be as in the theorem. Suppose that $I \subset A$ is a closed ideal and let $p$ be a nonnegative continuous function on $K$ such that given any $x \in F-p^{-1}(0)$ there is some $v \in I$ which satisfies $v(x) \neq 0$ and $|v(z)| \leqslant p(z)$ $(z \in K)$. Then given any $f \in C(F)$ satisfying $|f(s)| \leqslant p(s)(s \in F)$ there is an extension $\tilde{f} \in I$ of $f$ such that $|\tilde{f}(z)| \leqslant p(z)(z \in K)$. This sharpens [11, Theorem 20.12].

Corollary. Let $A$ be the disc algebra on the unit circle $T$ and let $p$ be a nonnegative continuous function on $T$ which does not vanish identically. The following are equivalent:

(i) The function $t \rightarrow \log p(t)$ is (Lebesgue) integrable on $T$.

(ii) Given any closed set $F \subset T$ of (Lebesgue) measure 0 and any continuous function $f \in C(F)$ satisfying $|f(s)| \leqslant p(s)(s \in F)$ there exists $\tilde{f} \in A$ which extends $f$ and satisfies $|\tilde{f}(z)| \leqslant p(z)(z \in T)$.

Proof. (ii) $\Rightarrow$ (i) follows from the fact that for any nonzero $g \in A$ the function $t \rightarrow \log |g(t)|$ is integrable [8]. To prove (i) $\Rightarrow$ (ii) let $p$ be a nonnegative continuous function on $T$ with $\log p$ integrable. By the F. and M. Riesz theorem [8] and by the Bishop theorem $A$ has the dominated extension property with respect to every closed set $F \subset T$ of measure 0 . So by our theorem it suffices to prove that given 
any $s \in T-p^{-1}(0)$ there is some $g \in A$ such that $g(s) \neq 0$ and $|g(z)|<p(z)$ $(z \in T)$. The following construction was suggested by D. Marshall. Writing $T-$ $p^{-1}(0)$ as a union of (at most countably many) pairwise disjoint open arcs and approximating $\log |p|$ on each of these arcs by a suitable continuously differentiable function one can get a nonnegative continuous function $q$ on $T$ such that $q<p$ on $T, \log q$ is integrable, $q^{-1}(0)=p^{-1}(0)$ and $q$ is continuously differentiable on $T-p^{-1}(0)$. Now the boundary function $g$ of the function

$$
z \rightarrow \exp \left(\frac{1}{2 \pi} \int_{0}^{2 \pi} \frac{e^{i \theta}+z}{e^{i \theta}-z} \log q\left(e^{i \theta}\right) d \theta\right) \quad(|z|<1)
$$

belongs to $A$ and satisfies $|g| \leqslant p$ on $T$ and $g^{-1}(0)=p^{-1}(0)$ [8]. Q.E.D.

The referee kindly suggested that the following two open problems could be mentioned.

1. In the case when $K=T$ and $A$ is the disc algebra on $T$ the condition (3) in the theorem can be replaced by the following weaker condition: There exists $g \in A$, $g \neq 0$, such that $|g(z)|<p(z)(z \in T)$. (This implies that $t \rightarrow \log p(t)$ is Lebesgue integrable on $T$ and the conclusion follows from the corollary.) Does this hold also in the case when $K$ is the closed unit disc and $A$ is the disc algebra on $K$ ? Simple examples show that this does not hold in general.

2. It is easy to see that (1) (implies and hence) is equivalent to the condition $\mu \in A^{\perp} \Rightarrow \mu_{F}=0$. Does the theorem hold if we replace the dominated extension property (1) by the condition $\mu \in A^{\perp} \Rightarrow \mu_{F} \in A^{\perp}$ and $C(F)$ by the restriction algebra $A \mid F$ ?

Acknowledgement. The author wishes to thank Professor Donald Marshall for a helpful discussion.

\section{REFERENCES}

1. E. M. Alfsen and B. Hirsberg, On dominated extensions in linear subspaces of $C_{c}(X)$, Pacific $J$. Math. 36 (1971), 567-584.

2. E. Bishop, A general Rudin-Carleson theorem, Proc. Amer. Math. Soc. 13 (1962), 140-143.

3. T. W. Gamelin, Restrictions of subspaces of $C(X)$, Trans. Amer. Math. Soc. 112 (1964), 278-286.

4. __ Uniform algebras, Prentice-Hall, Englewood Cliffs, N. J., 1969.

5. J. Globevnik, Analytic extensions and selections, Trans. Amer. Math. Soc. 254 (1979), 171-177.

6. P. Hag, A generalization of the Rudin-Carleson theorem, Proc. Amer. Math. Soc. 43 (1974), 341-344.

6a. __ Erratum to "A generalization of the Rudin-Carleson theorem", Proc. Amer. Math. Soc. 60 (1976), 377.

7.

8. K. Hoffman, Banach spaces of analytic functions, Prentice-Hall, Englewood Cliffs, N. J., 1962.

9. W. Roth, A general Rudin-Carleson theorem in Banach spaces, Pacific J. Math. 73 (1977), 197-213.

10. Z. Semadeni, Simultaneous extensions and projections in spaces of continuous functions, Lecture Notes, Univ. of Aarhus, 1965.

11. E. L. Stout, The theory of uniform algebras, Bogden and Quigley, Tarrytown-on-Hudson, N. Y., 1971.

Department of Mathematics, University of Washington, Seattle, Washmoton 98195

Current address: Institute of Mathematics, Physics and Mechanics, University of Ljubljana, Ljubljana, Yugoslavia 